\title{
Effect of Decannulation on Pharyngeal and Laryngeal Movement in Post-Stroke Tracheostomized Patients
}

\author{
Soo Jin Jung, M.D., Deog Young Kim, M.D., Ph.D., Yong Wook Kim, M.D., Ph.D., \\ Yoon Woo Koh, M.D., Ph.D. ${ }^{1}$, So Young Joo, M.D., Eun Sung Kim, OTR, M.S. ${ }^{2}$
}

Department and Research Institute of Rehabilitation Medicine, ${ }^{1}$ Otorhinolaryngology Medicine, ${ }^{2}$ Research Institute of Rehabilitation Medicine, Yonsei University College of Medicine, Seoul 120-752, Korea

Objective To investigate effects of tracheostomy tube on the movement of the hyoid bone and larynx during swallowing by quantitative analysis of videofluoroscopic swallowing study.

Method 19 adult stroke patients with tracheostomies, who met the criteria of decannulation participated. Serial videofluroscopic swallowing studies were done over 14 days before decannulation, within 24 hours before decannulation, within 24 hours after decannulation, and over 14 days after decannulation. The kinematic parameter such as pharyngeal transition time, stage transition duration, maximal hyoid bone movement, and maximal laryngeal prominence movement were obtained by 2-D quantitative analysis of videofluoroscopic swallowing study.

Results Pharyngeal transition time and stage transition duration were not significantly changed all the time. The maximal hyoid bone movement and maximal laryngeal prominence just after decannulation were improved significantly compared to just before decannulation $(\mathrm{p}<0.05)$, especially on vertical movement.

Conclusion The hypothesis that a tracheostomy tube disturbs the hyoid bone and laryngeal movement during swallowing may be supported by this study.

Key Words Tracheostomy, Decannulation, Hyoid bone, Laryngeal prominence, Movement

Received August 31, 2011; Accepted May 2, 2012

Corresponding author: Deog Young Kim

Department and Research Institute of Rehabilitation Medicine, Yonsei University College of Medicine, 50 Yonsei-ro, Seodaemun-gu, Seoul 120752, Korea

Tel: +82-2-2228-3714, Fax: +82-2-363-2795, E-mail: kimdy@yuhs.ac (c) This is an open-access article distributed under the terms of the Creative Commons Attribution Non-Commercial License (http:// creativecommons.org/licenses/by-nc/3.0) which permits unrestricted noncommercial use, distribution, and reproduction in any medium, provided the original work is properly cited

Copyright ( 2012 by Korean Academy of Rehabilitation Medicine

\section{INTRODUCTION}

A tracheostomy is performed for long term mechanical ventilation, central nervous system disorders, excessive respiratory secretion resolution, bypass surgery is required for upper airway obstruction. ${ }^{1,2}$ However, problems with a tracheostomy include: aspiration and dysphagia even though purpose of it's placement may be to prevent aspiration. ${ }^{3,4}$ Actually, aspiration is observed in 50 to $83 \%$ of patients with tracheostomy placement. ${ }^{5-7}$

Other complications linked to tracheostomy have been reported as hypokinesia and hypoesthesia which may 
occur in the swallowing mechanism. ${ }^{8}$ Dysphagia and aspiration may occur due to insufficient laryngeal elevation and desensitization of larynx, both of which can be caused by: an obstruction of upper esophagus due to the pressure of the tracheostomy tube and laryngeal hypoesthesia and hypokinesia due to tracheal mucosal injury or respiratory airflow abnormality. ${ }^{9}$ A hypothesis has been suggested in previous reports that a tracheostomy may affect pharyngeal structure movements, such as laryngeal elevation, and causes aspiration and dysphagia. Nevertheless, few studies have been conducted with a sufficient number of subjects, and moreover, quantitative analysis data has been insufficient as required..$^{10-16}$

Aspiration and dysphagia are known to occur during the pharyngeal stage in the swallowing process. It has recently been possible to make a quantitative analysis of kinematic indicators by videofluoroscopic swallowing studies (VFSS). ${ }^{17-20}$ In this study, VFSS were conducted on 19 patients before and after a tracheostomy procedure, and a two-dimensional quantitative analysis was made of kinematic indicators including the movements of the hyoid bone and the laryngeal prominence to investigate whether removal of the tracheostomy tube would cause changes in kinematic indicators, especially in the movements of the hyoid bone and the laryngeal prominence, during the swallowing process.

\section{MATERIALS AND METHODS}

\section{Subjects}

This study was conducted between March 2009 and April 2011 on stroke patients who required tracheostomies and were diagnosed with dysphagia by scoring less than or equal to 5 in 'American Speech-Language Hearing Association National Outcomes Measurements System Swallowing Scale (ASHA-NOMS),. ${ }^{21}$ Prior to decannulation, we checked tracheal patency with an otolaryngologist and investigated: whether mechanical ventilation could be stopped, whether respiration could be maintained for 48 hours or longer with the tube corked,

Table 1. General Characteristics of Subjects

\begin{tabular}{rrrlcccc}
\hline No. & Sex & $\begin{array}{l}\text { Age } \\
\text { (yr) }\end{array}$ & \multicolumn{1}{c}{ Diagnosis } & MMSE & $\begin{array}{c}\text { ASHA- } \\
\text { NORM } \\
\text { Scale }\end{array}$ & $\begin{array}{c}\text { Duration from } \\
\text { onset to trachestomy } \\
\text { (day) }\end{array}$ & $\begin{array}{c}\text { Duration from } \\
\text { onset to seal off } \\
\text { (day) }\end{array}$ \\
\hline 1 & M & 63 & Lt. Cbll hemisphere ICH & 26 & 5 & 0 & 288 \\
\hline 2 & M & 70 & Bilateral frontotemporal infarction & UC & 2 & 0 & 150 \\
\hline 3 & F & 43 & Lt. frontotemporal ICH & 3 & 2 & 14 & 79 \\
\hline 4 & M & 80 & Rt. thalamus ICH & 20 & 4 & 11 & 144 \\
\hline 5 & M & 60 & Rt. thalamus ICH & 18 & 2 & 11 & 84 \\
\hline 6 & M & 56 & Rt. basal ganglia ICH & UC & 5 & 0 & 115 \\
\hline 7 & M & 47 & Bilateral thalamus, Cbll ICH & 19 & 5 & 33 & 360 \\
\hline 8 & F & 78 & Rt. MCA territory infarction & 16 & 2 & 53 & 541 \\
\hline 9 & M & 49 & SAH & UC & 1 & 11 & 328 \\
\hline 10 & F & 49 & Rt. MCA territory infarction & UC & 2 & 0 & 82 \\
\hline 11 & F & 31 & Lt. frontal lobe ICH & 28 & 1 & 0 & 286 \\
\hline 12 & F & 59 & Rt. frontal lobe, basal ganglia ICH & UC & 1 & 1 & 197 \\
13 & M & 58 & Lt. frontal lobe, basal ganglia ICH & 14 & 3 & 3 & 74 \\
\hline 14 & M & 30 & SAH & 4 & 3 & 13 & 108 \\
\hline 15 & M & 28 & SAH & 4 & 1 & 9 & 107 \\
\hline 16 & F & 64 & SAH, subdural hematoma & UC & 5 & 0 & 450 \\
\hline 17 & M & 53 & Frontotemporal ICH & UC & 1 & 7 & 175 \\
\hline 18 & F & 43 & SAH & 11 & 1 & 14 & 89 \\
\hline 19 & M & 27 & Rt. BG ICH & 10 & 1 & 17 & 218 \\
\hline
\end{tabular}

UC: Uncheckable, ICH: Intracranial hemorrhage, SAH: Subarachnoidhemorrhage, ASHA-NOMS: American SpeechLanguage Hearing Association National Outcomes Measurements System Swallowing Scale, Cbll: Cerebellar 
and whether the patient could perform spontaneous sputum expectoration. ${ }^{8}$

A total of 19 patients were included in this study. The exclusion criteria included: patients who scored consider less than or equal to 9 in the JFK Coma Recovery Scale (JFK-CRS) Score ${ }^{22}$ (patients in vegetative states); patients that had a history of medical diseases (such as chronic obstructive pulmonary diseases, acute respiratory distress syndrome), and patients that had diseases which may lead to dysphgia (such as spinal cord injuries or spinal cord tumors, head and neck tumors, muscular diseases, hereditary diseases). The group of subjects had a mean age of 52.0. They were composed of 12 men and 7 women. Among the 19 patients, 16 had a hemorrhagic stroke and 3 had an cerebral infarction. The average length of time from tracheostomy to decannulation were performed 289.7 days and 460.6 days after symptom onset respectively. The ASHA-NOMS score averaged 2.8 which means degree of dysphagia.

\section{Methods}

Basic examinations: The period between symptom onset and tracheostomy placement and between symptom onset and decannulation were measured. Subjects were checked for cerebral infarction and cerebral hemorrhage in a brain CT or MRI, and took 'Korean Version of MiniMental State Exam (K-MMSE)' and JFK-CRS, ${ }^{22}$ in addition to ASHA-NOMS ${ }^{21}$ to gather basic information on dysphagia (Table 1).
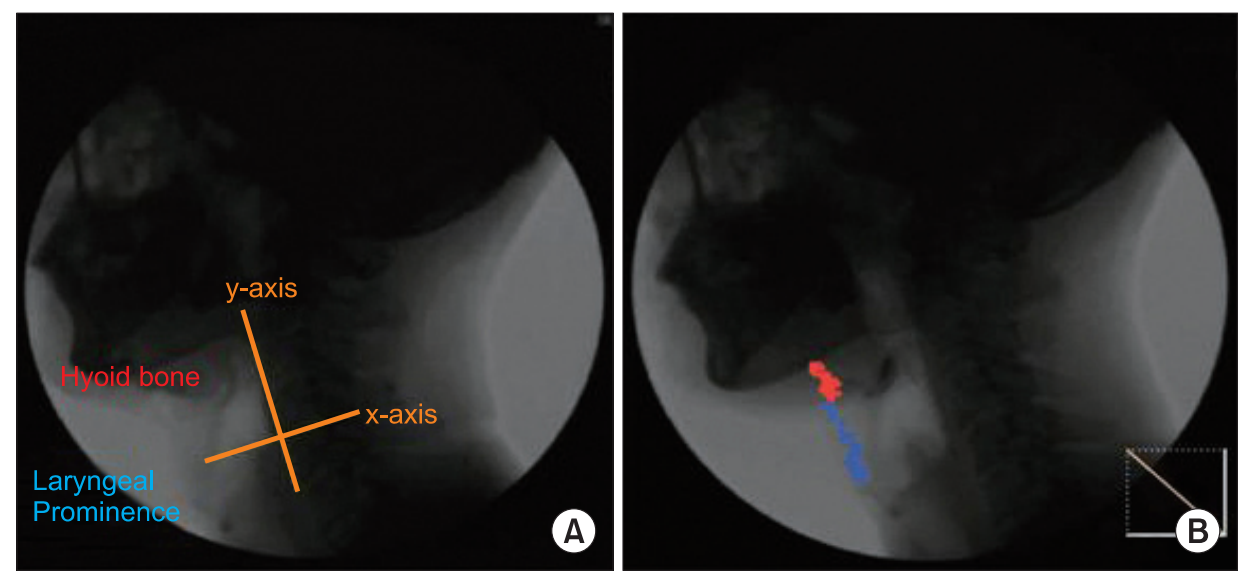

Fig. 2. Kinematic analysis using 2D graphic analysis program. (A) y-axis was defined as straight line connecting the antero-inferior border of the $\mathrm{C} 4$ vertebra to the antero-inferior border of the $\mathrm{C} 2$ vertebra. $\mathrm{x}$-axis was defined as straight perpendicular line to $\mathrm{y}$-axis crossing antero-inferior borderof the $\mathrm{C} 4$ vertebra. Red dot indicated anterior superior border of hyoid bone. Blue dot indicated laryngeal prominence. (B) The figure showed trajectory of hyoid bone movement (red spot), and that of laryngeal prominent movement (blue spot) during swallowing.

Fig. 1. Study design of experiment. VFSS: Videofluoroscopic swallow study, T0: Over 2 weeks before decannulation, T1: Within 24 hrs before decannulation, T2: Within 24 hrs after decannulation, T3: Over 2 weeks after decannulation.

(VFSS): VFSS before (T1), within 24 hours after (T2) and 2 weeks after (T3) decannulation. The results of 2 weeks before decannulation (T0) was compared with those of within 24 hours before decannulation (T1) in order to check out whether neurologic recovery was affected or a change occurred in the movements of the hyoid bone and the laryngeal prominence, and a comparison was made within 24 hours before decannulation (T1) and within 24 hours after decannulation (T2) to reveal a change that occurred immediately after decannulation. In addition, 2 weeks after decannulation (T3) was compared with within 24 hours before decannulation (T1) to prove out delay effects (Fig. 1). VFSS were performed 18 times in all and 3 times of each with 3 types of thickness (liquid, semisolid and solid) and 2 types of amount (5 cc and $15 \mathrm{cc}$ ). By use of a laser ranger finder DLE50 (Bosch, Stuttgart, Ger-

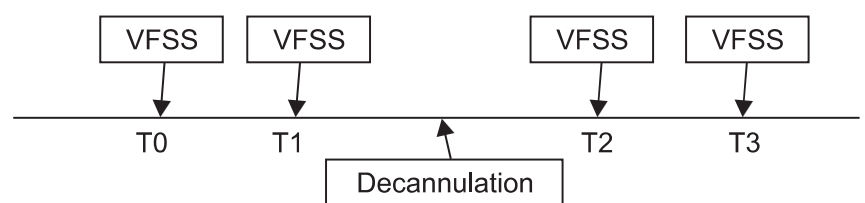


many), the radiation generator was so kept that it might be within $50 \mathrm{~cm}$ from the external auditory meatus and the deviation could be minimized. All the images of VFSS were recorded on DVDs at 30 frames per second by digital files.

Two-dimensional kinematic analysis: The images of videofluoroscopies were analyzed by the use of a twodimensional kinematic analysis program 'GraphClick (Arizona-Software, Phoenix, USA)'. The images were analyzed by one other than VFSS performers in order that errors could be minimized. The anterior region of the hyoid bone and the laryngeal prominence were marked separately on each frame. In accordance with the method of Logemann et al., ${ }^{19}$ the anteroinferior part of the fourth cervical vertebra was defined as the zero point, from where a line was drawn to the anteroinferior part of the second cervical vertebra. The line was defined as the y-axis. Another line, perpendicular to the y-axis, was defined as the x-axis. The results of VFSS, performed four times for each patient, were corrected in reference to the anterior length of the fourth cervical vertebra, which is a

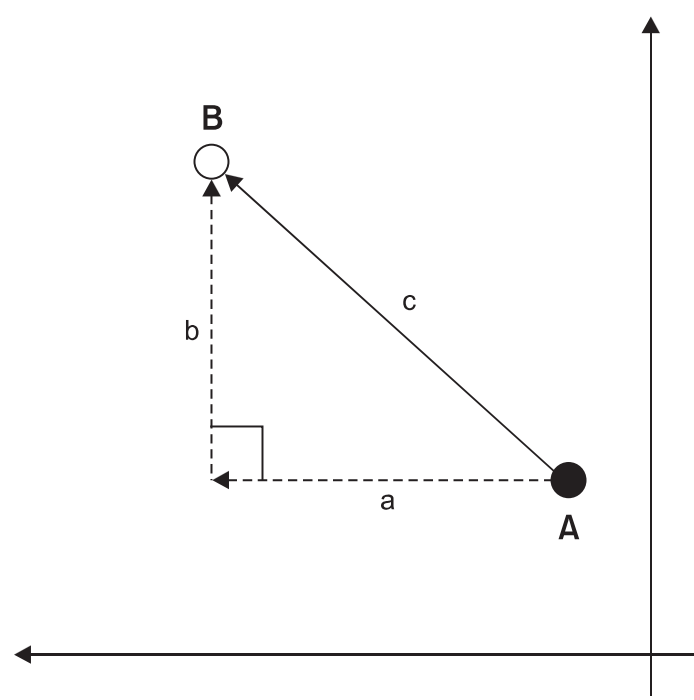

Fig. 3. Measurement of maximal, vertical, horizontal movement. A: Location at beginning, B: Location at maximal movement, a: Horizontal movement, b: Vertical movement, c: Total maximal movement. fixed length (Fig. 2). The maximal movements of the hyoid bone and the laryngeal prominence were calculated on the basis of $\mathrm{x}$ and $\mathrm{y}$-axes. Pharyngeal transition time was defined as the time it took for a bolus to pass through the upper esophageal sphincter via line between the mandibular ramus and the lingual base. Stage transition duration was defined as the time it took for the larynx to be lifted after the bolus head reached the line between the lingual base and the lower boundary of the mandible; the time was regarded as minus when the larynx was lifted before the head of a bolus reaches the line between the lingual base and the lower boundary of the mandible. ${ }^{23}$ A calculation was made of maximal hyoid movement ' $c$ ' on the basis of $\mathrm{x}$ and $\mathrm{y}$-coordinates worked out in regards to the position taken before hyoid movement (A) and the position where it was maximal displacement (B). Likewise, maximal laryngeal movement 'c' was calculated with $\mathrm{x}$ and $\mathrm{y}$-coordinates worked out as regards the position taken before laryngeal movement (A) and the position of maximal movement (B) (Fig. 3).

A statistical analysis was made of the kinematic differences among periods (T1, T2, T3) by use of the repeated ANOVA test, and inter-period differences (T1-T2, T2-T3 and T2-T4) were analyzed through the paired t-test. The data were finally verified with Bonfenoni correction. All data were analyzed by use of the 'Statistical Package for the Social Science (SPSS; version 17.0)' program and the significance level was defined as where the p-value was less than 0.05 .

\section{RESULTS}

\section{Quantitative comparison of kinematic data before decannulation}

In comparing pharyngeal transition time and stage transition duration, there were no statistically significant differences 2 weeks before, within 24 hours before, within 24 hours after and 2 weeks after decannulation (Table 2).

No statistically significant differences in the maximal, horizontal and vertical movements of the hyoid bone, 2

Table 2. Changes of Temporal Parameters after Decannulation

\begin{tabular}{lcccc}
\hline & T0 $(\mathbf{s e c})$ & T1 (sec) & T2 (sec) & T3 (sec) \\
\hline Pharyngeal transition time & $1.23 \pm 0.10$ & $1.30 \pm 0.10$ & $1.36 \pm 0.14$ & $1.16 \pm 0.07$ \\
Stage transition duration & $5.76 \pm 1.86$ & $2.38 \pm 0.67$ & $3.98 \pm 0.97$ & $2.81 \pm 0.61$ \\
\hline
\end{tabular}

Values are mean \pm standard error ${ }^{*} \mathrm{p}<0.05$ (paired t-test) 

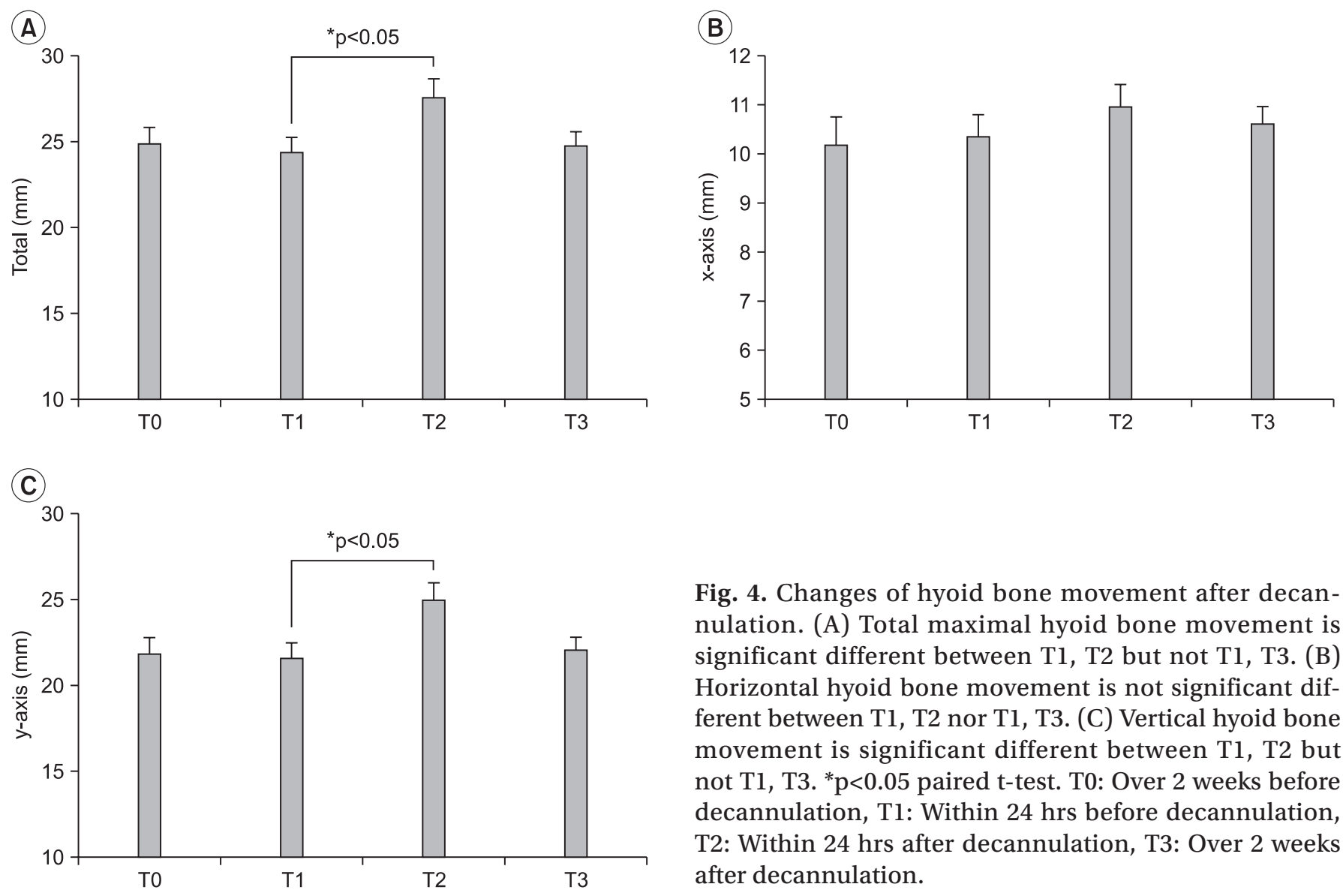

weeks before and within 24 hours before decannulation (Fig. 4). In the maximal, horizontal and vertical movements of the laryngeal prominence, statistically significant differences were not observed 2 weeks beforeand within 24 hours before decannulation (Fig. 5).

\section{Quantitative comparison of kinematic data after decannulation}

On the repeated ANOVA test conducted on the maximal and vertical movements of the hyoid bone, statistically significant differences were observed within 24 hours before, within 24 hours after and 2 weeks after decannulation ( $\mathrm{p}=0.001,0.007)$. In horizontal hyoid movement, however, there was no significant difference within 24 hours before, within 24 hours after and 2 weeks after decannulation $(\mathrm{p}=0.462)$. The horizontal and vertical movements of the laryngeal prominence showed statistically significant difference $(\mathrm{p}=0.003,0.003,0.002)$.

Maximal hyoid movement was calculated at $24.2 \pm 1.1$ within 24 hours before decannulation and at $27.5 \pm 1.2$ within 24 hours after decannulation, and thus a statisti-

Fig. 4. Changes of hyoid bone movement after decannulation. (A) Total maximal hyoid bone movement is significant different between T1, T2 but not T1, T3. (B) Horizontal hyoid bone movement is not significant different between T1, T2 nor T1, T3. (C) Vertical hyoid bone movement is significant different between $\mathrm{T} 1, \mathrm{~T} 2$ but not T1, T3. ${ }^{*}$ p $<0.05$ paired t-test. T0: Over 2 weeks before decannulation, T1: Within 24 hrs before decannulation, T2: Within 24 hrs after decannulation, T3: Over 2 weeks after decannulation.

cally significant increase was observed on the paired t-test ( $\mathrm{p}=0.006$ ). In horizontal movement, significant differences were not observed within 24 hours before and after decannulation. However, the vertical movement significantly increased from $21.5 \pm 1.0$ (within 24 hours before decannulation) to $24.9 \pm 11.5$ (within 24 hours after decannulation), ( $\mathrm{p}=0.002$ ) (Fig. 4).

Maximal laryngeal movement was calculated at $26.1 \pm 1.1$ within 24 hours before decannulation and at $31.9 \pm 1.324$ hours after it, and increased significantly in the paired $\mathrm{t}$-test $(\mathrm{p}=0.000)$. In horizontal movement, there was no significant difference at the two time points. On the other hand, the vertical movement significantly increased from $24.5 \pm 10.9$ to $30.3 \pm 1.3$ at respective timepoints ( $\mathrm{p}=0.000$ ) (Fig. 5).

Maximal laryngeal movement tended to decrease after decannulation, but it significantly increased 2 weeks after decannulation on the paired t-test $(26.1 \pm 1.1,29.6 \pm 1.1)$ $(p=0.001)$. The horizontal movement was calculated at $7.7 \pm 0.5$ immediately before decannulation and increased to $10.0 \pm 0.6$ after 2 weeks, showed a statistically significant 

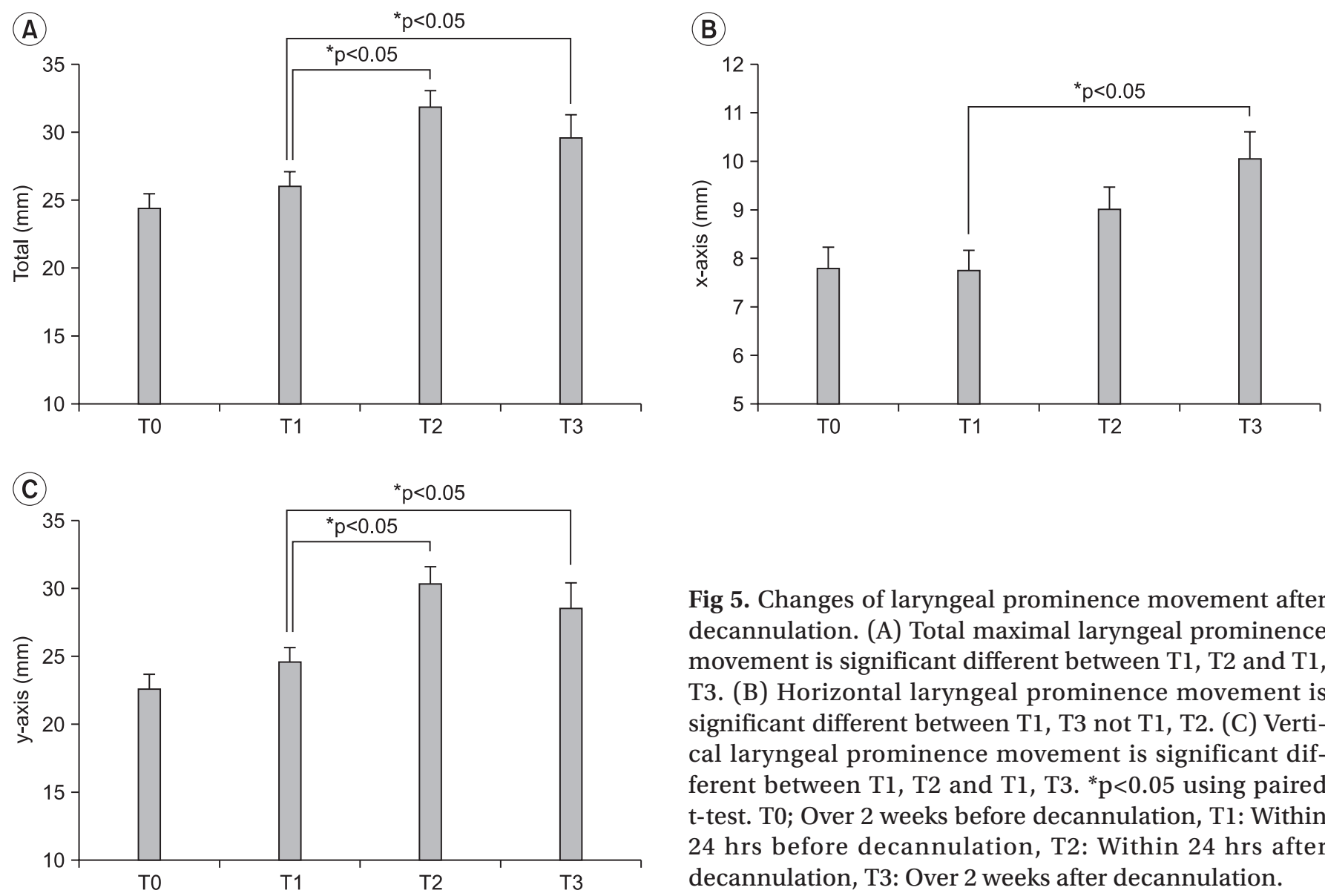

increase $(\mathrm{p}=0.000)$. Moreover, the vertical movement significantly increased from $8.5 \pm 1.9$ to $24.5 \pm 10.8(\mathrm{p}=0.016)$. In hyoid movement, any significant differences were not observed within 24 hours before decannulation, 2 weeks after decannulation (Fig. 3, 4).

Pharyngeal transition time and stage transition duration did not show any significant differences within 24 hours before, within 24 hours after and 2 weeks after decannulation (Table 2).

Correlation between severity of dysphagia and change of kinematic data

Significant correlations were not observed in the ASHANOMS scores measured within 24 hours before decannulation, pharyngeal transition time taken within 24 hours before and after it, and stage transition duration taken at the same time points. All indicators, relevant to hyoid and laryngeal movements, did not show significant correlations with severity of dysphagia.

Fig 5. Changes of laryngeal prominence movement after decannulation. (A) Total maximal laryngeal prominence movement is significant different between $\mathrm{T} 1, \mathrm{~T} 2$ and $\mathrm{T} 1$, T3. (B) Horizontal laryngeal prominence movement is significant different between T1, T3 not T1, T2. (C) Vertical laryngeal prominence movement is significant different between $\mathrm{T} 1, \mathrm{~T} 2$ and $\mathrm{T} 1, \mathrm{~T} 3 .{ }^{*} \mathrm{p}<0.05$ using paired t-test. T0; Over 2 weeks before decannulation, T1: Within $24 \mathrm{hrs}$ before decannulation, T2: Within $24 \mathrm{hrs}$ after decannulation, T3: Over 2 weeks after decannulation.

\section{DISCUSSION}

It has been reported that aspiration occurs in 50 to $83 \%$ of patients who underwent tracheostomies. ${ }^{11,24}$ Cha et al., ${ }^{25}$ who conducted VFSS on stroke patients having tracheostomy and ones who did not, reported that the two did not show significant differences during the oral stage and the laryngeal stage, but that asymptomatic aspiration occurred much more in patients who underwent tracheostomy.

The hyoid bone protects the airway, in order to prevent aspiration in a swallowing process, the suprahyoid muscle contracts anterosuperiorly and covers the epiglottis. In case of the decrease of hyoid bone movement, it heightens the risk of aspiration, disturbes the relaxation of cricopharyngeous muscle, and retards the transit of boluses. $^{26,27}$

Bonanno ${ }^{10}$ researched into hyoid lifts before and after tracheostomy in 3 patients, and reported that the tracheostomy tube hindered effective deglutition because it fixed the larynx to the tissue around the neck, so dis- 
turbed elevation of hyoid-mandibular complex, anterior displacing of the larynx, and hindered the relaxation of cricopharyngeous muscle. Also consider long term VFSS on patients was conducted by Elpern et al. ${ }^{28}$ showed aspirations after undergoing tracheostomies and ones who did not, and reported that inactive laryngeal elevations were more frequent in patients who showed aspirations, which implies that inactive laryngeal elevation is a principal factor of aspiration that occurs after tracheostomy. Ding and Logemann ${ }^{11}$ reported that inactive laryngeal elevation and abnormal aspiration increased more significantly when the cuff of the tracheostomy tube was inflating than when it was deflating. Furthermore, a hypothesis has been formulated in many reports that the tracheostomy tube may inhibit laryngeal elevation. ${ }^{29,30}$

On the contrary to the a fore mentioned, Terk et al., ${ }^{15}$ who made a quantitative analysis of pharynx-hyoid distance and maximal hyoid movement in 7 patients, and represented that there were no significant differences between patients who were having tracheostomy tubes placed in their tracheas and ones who did not.

In this quantitative analysis study, the use of kinematic indicators during the laryngeal stage, showed that maximal and vertical hyoid movements and laryngeal movement significantly increased immediately after decannulation compared to before it. For reference, horizontal hyoid movement and laryngeal movement did not show significant differences. Laryngeal movement increased significantly 2 weeks after decannulation. These results were similar to those of previous studies. ${ }^{4,10,29,30}$ What is remarkable is that this study and that of Terk et al. ${ }^{15}$ showed different results though they were similar in measurement. Possible reasons are as follows: the study of Terk et al. ${ }^{15}$ was a limit to small number of subjects, and this study was conducted on patients with dysphagia, whereas their study on patients who were able to take oral foods and had no dysphagia in spite of tracheostomy.

The results of this study support the hypothesis that the tracheostomy tube fixes the larynx to the tissue around the neck and hinders laryngeal movements and hyoid and laryngeal movements during the normal swallowing process, but Logemann et al. ${ }^{31}$ reported that when the upper esophageal sphincter opened, maximal laryngeal and hyoid lifts could be improved just by the tracheostomy tube's being stopped up by hand. To sum them up, it appears that the tracheotomy tube directly disturbes laryngeal movements.

Goldsmith $^{32}$ reported that the tracheostomy tube itself is fixed the larynx, could not form sublingual pressure, and caused delayed effects relevant to muscular damage and disuse atrophy. In this study VFSS, conducted immediately after and 2 weeks after decannulation, showed that changes occurred in most movements excluding horizontal laryngeal movement, and that delayed effects were observed partially.

In addition, it is known that the tracheostomy tube causes disturbance of oral movement, changes in anatomical structure, decrease of sensation, the obstruction of airway obstruction related to the traheostomy and impairment of motor coordination, ${ }^{12}$ and that it changes pharyngeal aerodynamics, precludes positive subglottic air pressure, and disturbed the larynx defense reflex. ${ }^{18,31,33}$ The tracheostomy tube cuff inflation is not the absolute solution to aspiration, ${ }^{13}$ an increase in cuff pressure heightens swallowing pressure, which may end in tissue necrosis and tracheal wall injury. ${ }^{35}$ Accordingly, there is the need to conduct future research into the influence of tracheostomy on dysphagia and aspiration.

In this study there was a limit to sample size. Thus, it future studies should be conducted with a sufficient number of subjects. Also there were no significant differences in all the indicators checked 2 weeks before and immediately before decannulation, but the problem is that the comparison was limited to kinematic indicators and the influences of various variables might not be ruled out completely. In this regard, a randomized and controlled study needs to be conductedso that more accurate results may be produced afterward.

\section{CONCLUSION}

In this quantitative analysis study using of kinematic indicators that indicated during the pharyngeal stage. The results showed that after decannulation, laryngeal and hyoid movements were improved in the swallowing process, which may be admissible as evidence to support the hypothesis that tracheostomy may disturb laryngeal and hyoid movements. 


\section{ACKNOWLEDGEMENTS}

This work was supported by Mid-career Researcher Program through NRF grant funded by the MEST (No. 20120000155).

\section{REFERENCES}

1. Frutos-Vivar F, Esteban A, Apezteguia C, Anzueto A, Nightingale P, Gonzalez M, Soto L, Rodrigo C, Raad J, David CM, et al. Outcome of mechanically ventilated patients who require a tracheostomy. Crit Care Med 2005; 33: 290-298

2. Kollef MH, Ahrens TS, Shannon W. Clinical predictors and outcomes for patients requiring tracheostomy in the intensive care unit. Crit Care Med 1999; 27: 17141720

3. Nash M. Swallowing problems in the tracheostomized patient. Otolaryngol Clin North Am 1988; 21: 701-709

4. McCulloch TM, Jaffe D, Hoffman HT. Diseases and operation of head and neck structures affecting swallowing. In: Perlman AL, Schulze-Delriew KS, Editors. Deglutition and its disorders, 1st ed, San Diego: Singular Publishing Group Inc, 1997, 343-381

5. Cameron JL, Reynolds J, Zuidema GD. Aspiration in patients with tracheotomies. Surg Gynecol Obstet 1973; 136: 68-70

6. Elpern EH, Scott MG, Petro L, Ries MH. Pulmonary aspiration in mechanically ventilated patients with tracheostomies. Chest 1994; 105: 563-566

7. Tolep K, Getch CL, Criner GJ. Swallowing dysfunction in patients receiving prolonged mechanical ventilation. Chest 1996; 109: 167-172

8. O'Connor HH, White AC. Tracheostomy decannulation. Respir Care 2010; 55: 1076-1081

9. Smith-Hammond CA, Goldstein LB. Cough and aspiration of food and liquids due to oral-pharyngeal dysphagia: ACCP evidence-based clinical practice guidelines. Chest 2006;129 Suppl 1: S154-168

10. Bonanno PC. Swallowing dysfunction after tracheostomy. Ann Surg 1971; 174: 29-33

11. Ding R, Logemann JA. Swallow physiology in patients with trach cuff inflated or deflated: a retrospective study. Head Neck 2005; 27: 809-813

12. DeVita MA, Spierer-Rundback L. Swallowing disorders in patients with prolonged orotracheal intuba- tion or tracheostomy tubes. Crit Care Med 1990; 18: 1328-1330

13. Higgins DM, Maclean JC. Dysphagia in the patient with a tracheostomy: six cases of inappropriate cuff deflation or removal. Heart Lung 1997; 26: 215-220

14. Suiter DM, McCullough GH, Powell PW. Effects of cuff deflation and one-way tracheostomy speaking valve placement on swallow physiology. Dysphagia 2003; 18: 284-292

15. Terk AR, Leder SB, Burrell MI. Hyoid bone and laryngeal movement dependent upon presence of a tracheotomy tube. Dysphagia 2007; 22: 89-93

16. Gross RD, Mahlmann J, Grayhack JP. Physiologic effects of open and closed tracheostomy tubes on the pharyngeal swallow. Ann Otol Rhinol Laryngol 2003; 112: 143-152

17. Hiiemae KM, Palmer JB, Medicis SW, Hegener J, Jackson BS, Lieberman DE. Hyoid and tongue surface movements in speaking and eating. Arch Oral Biol 2002; 47: 11-27

18. Ishida R, Palmer JB, Hiiemae KM. Hyoid motion during swallowing: factors affecting forward and upward displacement. Dysphagia 2002; 17: 262-272

19. Logemann JA, Pauloski BR, Rademaker AW, Colangelo LA, Kahrilas PJ, Smith CH. Temporal and biomechanical characteristics of oropharyngeal swallow in younger and older men. J Speech Lang Hear Res 2000; 43: 1264-1274

20. Kim SJ, Lee KJ. Comparison of the hyoid movement during the pharyngolaryngeal swallowing at the healthy young and old person. J Korean Acad Rehab Med 2006; 30: 485-490

21. Wesling M, Brady S, Jensen M, Nickell M, Statkus D, Escobar N. Dysphagia outcomes in patients with brain tumors undergoing inpatient rehabilitation. Dysphagia 2003; 18: 203-210

22. Giacino JT, Kalmar K. Comarecoveryscale-revisedadministration and scoring guidelines, 1st ed, New Jersey: Solaris Health System, 2004, 1-15

23. Kendall KA, McKenzie S, Leonard RJ, Goncalves MI, Walker A. Timing of events in normal swallowing: a videofluoroscopic study. Dysphagia 2000; 15: 74-83

24. Muz J, Mathog RH, Nelson R, Jones LA Jr. Aspiration in patients with head and neck cancer and tracheostomy. Am J Otolaryngol 1989; 10: 282-286

25. Cha DY, Yang HS, Noh JY, Lee SY, Choi JY, Kim SN, 
Park YO. Effect of tracheostomy tube on swallowing in patients with stroke. J Korean Acad Rehab Med 2010; 34: 128-133

26. Dodds WJ, Stewart ET, Logemann JA. Physiology and radiology of the normal oral and pharyngeal phases of swallowing. AJR Am J Roentgenol 1990; 154: 953-963

27. Dodds WJ, Logemann JA, Stewart ET. Radiologic assessment of abnormal oral and pharyngeal phases of swallowing. AJR Am J Roentgenol 1990; 154: 965-974

28. Elpern EH, Scott MG, Petro L, Ries MH. Pulmonary aspiration in mechanically ventilated patients with tracheostomies. Chest 1994; 105: 563-566

29. Logemann JA. Evaluation and treatment of swallowing disorders, 1st ed, San diego: College-Hill Press Inc,
1983, 38-50

30. Buchwalter JA, Sasaki CT. Effect of tracheotomy on laryngeal function. Otolaryngol Clin North Am 1984; 17: 41-48

31. Logemann JA, Pauloski BR, Colangelo L. Light digital occlusion of the tracheostomy tube: a pilot study of effects on aspiration and biomechanics of the swallow. Head Neck 1998; 20: 52-57

32. Goldsmith T. Evaluation and treatment of swallowing disorders following endotracheal intubation and tracheostomy. Int Anesthesiol Clin 2000; 38: 219-242

33. Kronenberger MB, Meyers AD. Dysphagia following head and neck cancer surgery. Dysphagia 1994; 9: 236-244 